\title{
Neutrino beams as a probe of the nuclear isospin and spin-isospin excitations
}

\author{
R. Lazauskas ${ }^{\text {a* }}$, C. Volpe ${ }^{\text {a† }}$ \\ anstitut de Physique Nucléaire, F-91406 Orsay cedex, France \\ We explore the possibility of performing nuclear structure studies using low energy \\ neutrino beams. In particular, low energy beta-beams and conventional sources (muon \\ decay-at-rest) are considered. We present results on the total charged-current as well as \\ flux-averaged cross sections associated to electron (anti)-neutrino scattering on oxygen, \\ iron, molybdenum and lead, as typical examples. It is shown that by using neutrinos from \\ low energy beta-beams, information on forbidden states, in particular the spin-dipole, \\ could be extracted.
}

\section{Introduction}

Nuclear isospin and spin-isospin excitations play a crucial role in weak processes. A precise description of these transitions is necessary to progress on open issues in high energy physics and in astrophysics. Searches for processes beyond the Standard Model, such as neutrinoless double beta-decay, require a detailed knowledge of the isospin and spin-isospin nuclear response. In particular, beta-decay [1], muon capture [2.3] the $2 \nu$ double-beta decay [4] as well as charge-exchange studies [5,6,7] are essential to constrain neutrinoless double-beta decay predictions; while neutrino-nucleus has been pointed out recently as a new constraint [8]. Beta decay is one of the key ingredients of the nucleosynthesis of heavy elements (see e.g. [9]10]). The role of neutrino-nucleus interactions is emphasized in [11] for neutrino-nucleosynthesis and for the r-process [12,13]14]15]. Such processes are also an essential ingredient to determine the response of neutrino detectors which can serve as core-collapse Supernova observatories.

The Isobaric Analogue States (IAS) as well as the Gamow-Teller (GT) giant resonances represent the most studied cases. Information on the associated nuclear transitions is essentially obtained through beta-decay or charge-exchange reactions. A satisfactory description of these excitations is now achieved using microscopic approaches: the shell model or the random-phase-approximation (RPA) and its variants. The effective interactions associated with these models have been extensively discussed and some of their parameters have been determined using the allowed transitions as a constraint (see e.g. [16]). However, one is still not able to explain why the measured GT transition amplitudes are always larger than the calculated ones. This is usually corrected by using an effective axial-vector coupling constant (the "quenching problem" [17],18]).

*E-mail: lazauskas@lpsc.in2p3.fr

${ }^{\dagger}$ E-mail: volpe@ipno.in2p3.fr 
Little is still known for the states of higher multipolarity, such as the spin-dipole or the higher multipoles. Their importance has been underlined in astrophysics (see e.g. [13]) and is well established for neutrinoless double-beta decay (see e.g. [19,20]). Using sumrules, one can relate the spin-dipole strength distribution to the neutron skin thickness defined as the difference between the root mean square radii of the proton and neutron distributions. This is an useful information to establish the nuclear matter equation of state of neutron stars 21. Some information on these states has been extracted thanks to various weak and hadronic probes. A systematic study of muon capture is performed in 22 and it is shown that no quenching of the forbidden states is required to account for the total capture rates. On the other hand, experiments with $(p, n)$ and $\left({ }^{3} \mathrm{He}, \mathrm{t}\right)$ have been realized [23]. Calculations based on RPA overestimate the experimental results in the Gamow-Teller region, while they underestimate them in the 30-60 MeV energy range [21]. Therefore if quenching of the forbidden states is necessary is an open issue. This question could be settled if more experimental information was available. Furthermore the latter could be exploited to improve the parametrization of the phenomenological interactions used in microscopic approaches.

Neutrino-nucleus interactions can probe different spin-isospin and isospin states, depending on the neutrino energy. While neutrinos in the tens of $\mathrm{MeV}$, such as those produced by the sun, essentially explore the IAS and GT excited states, those having several tens to hundreds $\mathrm{MeV}$, e.g. from core-collapse supernovea or from accelerators, also probe the spin-dipole and higher multipoles. These measurements are in most cases inclusive. However, different pieces of information can be obtained by using either sources where the energy of the neutrinos can be tuned or complementary probes. So far experiments are scarce, and include: a measurement on deuteron using reactor neutrinos [24], one on iron [25] and several on carbon with conventional sources (muon decay-at-rest or decay-in-flight) [26]. Therefore for the numerous applications, one relies on the theoretical predictions available in the literature and based on different approaches. The calculations generally agree for neutrinos in the ten of $\mathrm{MeV}$ where the response is dominated by the IAS and the GT transitions. However, significant discrepancies appear at higher energies (see e.g. [27,28] for carbon and [30,31,32,33] for lead). The origin of these disagreements is not yet clear and can be due to a different choice in the nuclear structure description (e.g. the continuum treatment, the choice of the effective forces, or the correlations included).

Systematic neutrino-nucleus interaction measurements could be an ideal tool to explore the weak nuclear response. At present, new experiments on various nuclei are being proposed with a new facility using muon decay-at-rest [34. Another possibility could be offered by beta-beams. This is a new method to produce pure and well known electron neutrino beams, exploiting the beta-decay of boosted radioactive ions [35]. The idea of establishing a low energy beta-beam facility has been first proposed in [36]. Its potential has been discussed in [36,37,38] for nuclear structure studies, in [36,39] for core-collapse supernova physics, and in [40,8,41,42,43,44] for the study of fundamental interactions. (For a review on the low, medium and high energy scenarios as well as their physics potential see [45]). An analysis of how neutrino spectral shapes change at low-energy beta-beams depending on the detector geometry and different locations within the same detector is made in [46], while the possibility of exploiting low-energy neutrinos at off-axis from a standard beta-beam is proposed in [47]. 
In this paper, we consider low energy beta-beams as a tool to study the isospin and spinisospin nuclear response. This feature has been first underlined in [36] and explored for the neutrino-lead reaction in 38 . Here we perform microscopic calculations of chargedcurrent neutrino-nucleus interactions. The nuclear amplitudes are obtained using the mean field approach including pairing correlations and the random-phase-approximation among quasi-particles. We present the total neutrino-nucleus cross sections as well as the contribution of the various multipoles and discuss how their importance evolve, as a function of neutrino energy. We give the flux-averaged cross sections associated to low energy beta-beams and to conventional sources (muon decay-at-rest), and compare the latter to previous calculations, when available. Results are given for oxygen, iron, molybdenum and lead taken as sample nuclei. These nuclei present particular interest for neutrino detectors and supernova observatories, currently used or under study. In particular, oxygen is used in water Cherenkov detectors, like Super-Kamiokande, the next-generation UNO [48, MEMPHYS [49] and Hyper-Kamiokande [50]. An iron-based detector was considered in [34]; while molybdenum- [51] and lead [52] ones are being studied. Note that the contribution of the different multipoles to the neutral current neutrino-lead cross section was studied theoretically in Ref.[53].

The paper is organized as follows. The theoretical aspects are described in Section II. Results on the isospin and spin-isospin states contributing to the cross sections as well as on the total and the flux-averaged cross sections are presented in Section III. Conclusions are drawn in Section IV.

\section{Calculations}

The total cross section for the charged current neutrino-nucleus scattering process $\nu_{l}\left(\bar{\nu}_{l}\right)+{ }^{A}$ $X \rightarrow l+{ }^{A} Y$ is given by [54]

$\sigma\left(E_{\nu}\right)=-i \int d^{3} k_{l} \delta\left(E_{l}+E_{f}-E_{\nu}-E_{i}\right)\left|\left\langle l\left(\vec{k}_{l}\right) ; f\left|H_{e f f}\right| \nu_{l}\left(\vec{k}_{\nu}\right) ; i\right\rangle\right|^{2}$,

where $E_{f}\left(E_{i}\right)$ is the energy of the final (initial) nuclear state, $E_{\nu}\left(\vec{k}_{\nu}\right)$ is the incident neutrino energy (momentum) and $E_{l}\left(\vec{k}_{l}\right)$ is the outgoing lepton energy (momentum). The effective single-particle hamiltonian $H_{\text {eff }}$ is derived by carrying out the Foldy-Wouthuysen (FW) transformation for the nucleon weak current operator and retaining terms up to $O(|\mathbf{q}| / M)$ (M is the nucleon mass and $\mathbf{q}$ is the momentum transfer). After performing a multipole expansion of the weak current, the differential cross section for the transition between nuclear states with angular momenta $J_{i}$ and $J_{f}$ is given by:

$\frac{d \sigma\left(E_{\nu}\right)}{d \Omega}=\frac{G^{2} \cos ^{2} \theta_{C} E_{l} k_{l}}{\pi} \frac{1}{2 J_{i}+1}\left(\sum_{J=0}^{\infty} \sigma_{C L}^{J}+\sum_{J=1}^{\infty} \sigma_{T}^{J}\right)$

where $G \cos \theta_{C}$ is the weak coupling constant. The $\sigma_{C L}^{J}$ and $\sigma_{T}^{J}$ represent the longitudinal and traverse response of the system to the external field. According to [54] these can be expressed as follows:

$$
\begin{aligned}
\sigma_{C L}^{J}= & (1+\widehat{v} \cdot \beta)\left|\left\langle J_{f}\left\|\widehat{\mathcal{M}}_{J}\right\| J_{i}\right\rangle\right|^{2}+[1-\widehat{v} \cdot \beta+2(\widehat{v} \cdot \widehat{q})(\widehat{q} \cdot \beta)]\left|\left\langle J_{f}\left\|\widehat{\mathcal{L}}_{J}\right\| J_{i}\right\rangle\right|^{2} \\
& -[\widehat{q} \cdot(\widehat{v}+\beta)] 2 \operatorname{Re}\left\langle J_{f}\left\|\widehat{\mathcal{L}}_{J}\right\| J_{i}\right\rangle\left\langle J_{f}\left\|\widehat{\mathcal{M}}_{J}\right\| J_{i}\right\rangle^{*}
\end{aligned}
$$




$$
\begin{aligned}
\sigma_{T}^{J}= & {[1-(\widehat{v} \cdot \widehat{q})(\widehat{q} \cdot \beta)]\left(\left|\left\langle J_{f}\left\|\widehat{\mathcal{J}}_{J}^{\text {mag }}\right\| J_{i}\right\rangle\right|^{2}+\left|\left\langle J_{f}\left\|\widehat{\mathcal{J}}_{J}^{e l}\right\| J_{i}\right\rangle\right|^{2}\right) } \\
& \pm[\widehat{q} \cdot(\widehat{v}-\beta)] 2 \operatorname{Re}\left\langle J_{f}\left\|\widehat{\mathcal{J}}_{J}^{\text {mag }}\right\| J_{i}\right\rangle\left\langle J_{f}\left\|\widehat{\mathcal{J}}_{J}^{e l}\right\| J_{i}\right\rangle^{*}
\end{aligned}
$$

where $\widehat{v}=\vec{k}_{\nu} / E_{\nu}$ is a unit vector defining the incoming (anti)neutrino flux $; \beta=\vec{k}_{l} / E_{l}$. $\widehat{\mathcal{M}}_{J}, \widehat{\mathcal{L}}_{J}, \widehat{\mathcal{J}}_{J}^{e l}$ and $\widehat{\mathcal{J}}_{J}^{\text {mag }}$ are the charge, longitudinal, traverse electric and traverse magnetic multipole operators, respectively. The plus or minus signs correspond to neutrino or antineutrino scattering respectively. The corresponding matrix elements defined for singleparticle transitions can be found in [54].

A correction to (2) must be introduced to account for the distortion of the outgoing lepton wave function due to the Coulomb field of the daughter nucleus. While the Fermi function $F\left(Z_{f}, E_{l}\right)$ accounts well for it at low neutrino energies, the modified "Effective Momentum Approximation" (EMA) offers a good procedure at high neutrino energies [55]. Here we follow the prescription already used in previous calculations, where the Fermi function is taken until the corresponding corrections give a larger cross section than those obtained with the EMA approximation.

Flux-averaged cross sections are the measured observables. These are obtained by folding the energy dependent cross section (2) with the neutrino flux $\phi\left(E_{\nu}\right)$ that depends on the specific neutrino source and the target geometry

$\langle\sigma\rangle_{\phi}=\int d E_{\nu} \sigma\left(E_{\nu}\right) \phi\left(E_{\nu}\right)$.

The transition matrix elements entering in Eqs. (3-5i) are calculated within microscopic approaches. The starting point is a Hartree-Fock (HF) calculation for the ground-state of the nucleus, performed in coordinate space by using the Skyrme-type effective interactions. The HF procedure determines the mean-field and single-particle (s.p.) occupied levels. The unoccupied levels are obtained by diagonalizing the HF mean-field using a harmonic oscillator basis. Therefore, the continuum part of the s.p. spectrum is discretized and discrete particle-hole (ph) configurations coupled to a given $J^{\pi}$ are used as a basis, in order to cast the RPA equations in the matrix form. This RPA calculation is self-consistent since the residual interaction among ph states is derived from the same Skyrme force as the one used to produce the mean field.

When necessary, to go beyond the closed-shell approximation for the ground state, pairing correlations are taken into account in the HF+BCS approximation. Constant pairing gaps $\Delta_{p}$ and $\Delta_{n}$ for protons and neutrons are introduced. On top of the HF+BCS calculation, the QRPA matrix equations can be written with a procedure which parallels what was described above, with the two-quasiparticle (2qp) configurations replacing the ph ones. We do not present here the details of the QRPA formalism, which can be found in the literature. We simply note that the particle-particle matrix elements are here renormalized by means of a parameter $g_{\mathrm{pp}}$ that has been chosen to be smaller than 1 (typically 0.7 ) to avoid the well-known ground-state instabilities.

For each multipolarity, every eigenstate of the RPA or QRPA equations is characterized by its $X^{f}$ and $Y^{f}$ amplitudes and the transition matrix element for a generic operator 


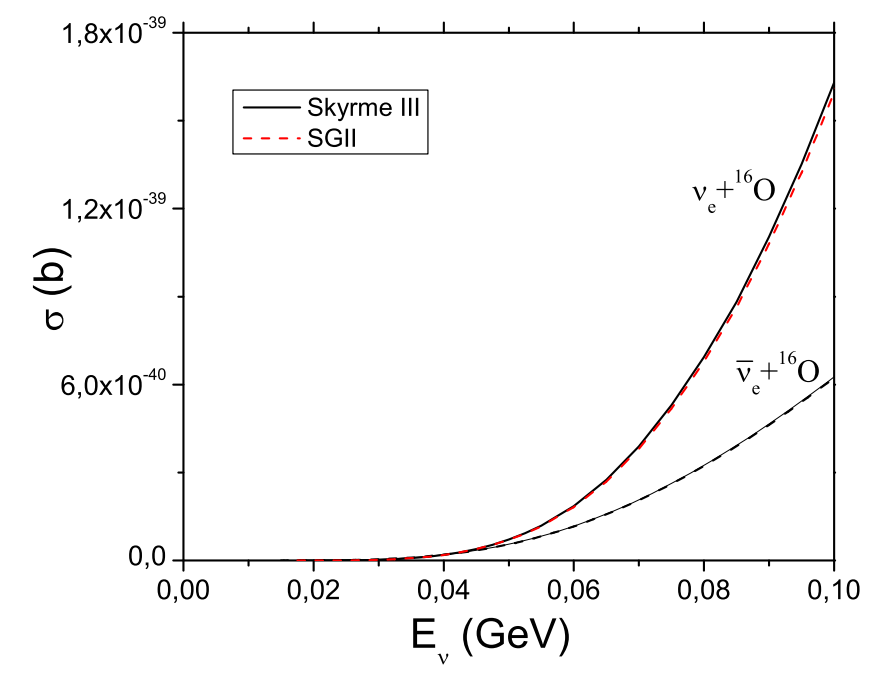

Figure 1. Total neutrino- ${ }^{16} \mathrm{O}$ cross section as a function of neutrino energy. Results obtained with the SGII and Skyrme III forces are shown.

$\hat{O}(k)$ is written as

$\left\langle J_{f}\left|\sum_{k} \hat{O}(k)\right| J_{i}\right\rangle=\sum_{\alpha, \beta}\left\langle\alpha\left|\sum_{k} \hat{O}(k)\right| \beta\right\rangle\left(X_{\alpha \beta}^{f} u_{\alpha} v_{\beta}+Y_{\alpha \beta}^{f} v_{\alpha} u_{\beta}\right)$,

where $\alpha$ and $\beta$ label a given ph or 2qp states, $u$ and $v$ are the BCS occupation amplitudes (which reduce to 1 and 0 in the HF-RPA case) and $\left\langle\alpha\left|\sum_{k} \hat{O}(k)\right| \beta\right\rangle$ are single-particle matrix elements.

\section{Results and discussion}

The calculations we show are performed using the Skyrme force. We have checked that the use of the SGII force does not change the results significantly (Figure 1). The value of $g_{p p}=0.7$ is used, but the results are stable against changes of this parameter (Figure 2). All states up to $J^{\pi}=5^{ \pm}$are included. We present results for four nuclei as typical examples, namely ${ }^{16} \mathrm{O},{ }^{56} \mathrm{Fe},{ }^{100} \mathrm{Mo}$ and ${ }^{208} \mathrm{~Pb}$. The total (anti-)neutrino cross sections are given in Table 1 for the four nuclei considered.

We have compared our results to previous calculations, when available, either on the total cross sections as a function of neutrino energy, or on the flux-averaged cross sections associated to electron neutrinos from decay-at-rest muons. Our results are in reasonable agreement with those of Refs. [28,33,29] for oxygen, of Ref. [31,33,57,58] for iron and of Ref.[31,33,59] for lead. As far as the comparison with measurements is concerned, the only available is the neutrino-iron cross section. The muon decay-at-rest flux averaged 


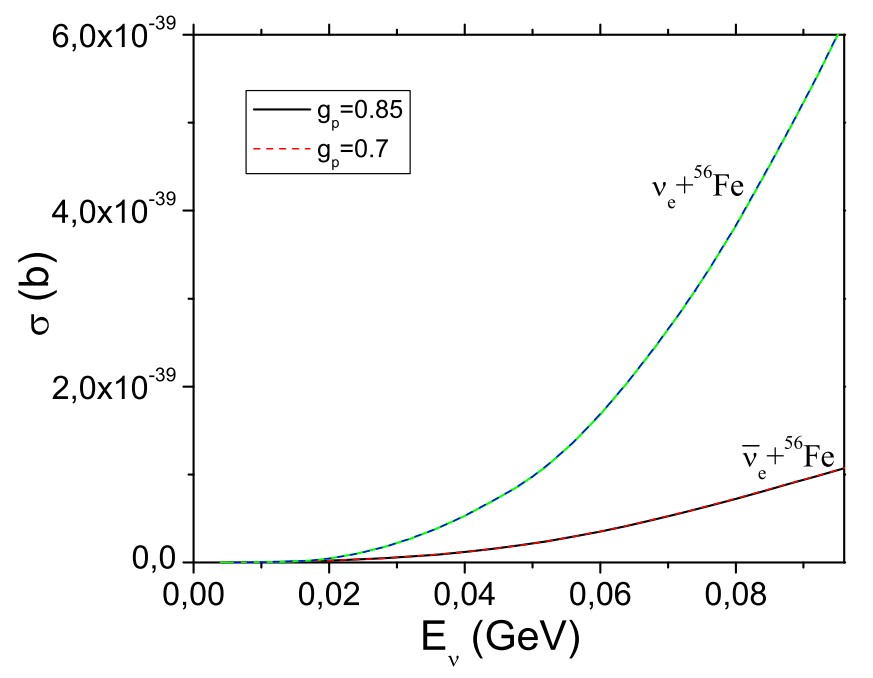

Figure 2. Total (anti)neutrino- ${ }^{16}$ Fe cross section as a function of neutrino energy. Results obtained for two values of the $g_{p p}$ parameter renormalizing the particle-particle interaction in the QRPA calculations (see text) are given.

cross section is $256 \pm 108 \pm 43\left(10^{-42} \mathrm{~cm}^{2}\right)$. Our result, $35210^{-42} \mathrm{~cm}^{2}$, is in agreement with the experimental value.

The contribution of the different multipoles as a function of the neutrino energy are shown in Figure 3 for iron, as an example, both for positive and negative parity states. Below $40 \mathrm{MeV}$ the GT $J^{\pi}=1^{+}$transitions dominate the cross section. At $60 \mathrm{MeV}$ the $J^{\pi}=1^{-}, 2^{-}$become important as well. Beyond $80 \mathrm{MeV}$ all states contribute.

The details on the neutrino flux associated to low-energy beta-beams are given in 37] (Fig. 4). Following a preliminary feasibility study, we assume, as in [4142], that the boosted ions are stored in a storage ring which has $1885 \mathrm{~m}$ total length and $678 \mathrm{~m}$ straight sections, and that the detectors are located at $10 \mathrm{~m}$ from the storage ring [56]. We consider fully efficient cylindrical detectors as in [37], namely $R=1.5 \mathrm{~m}$ (radius) and $h=4.5 \mathrm{~m}$ (depth) for metals and $R=4.5 \mathrm{~m}, h=15 \mathrm{~m}$ water Cherenkov detectors for oxygen. The neutrino flux corresponding to conventional neutrino sources, i.e. muon decay-at-rest (DAR) is given by the well known Michel spectrum. As seen in Figure 4, the latter has quite similar shape to a low energy beta-beam with $\gamma=10$. Note that in principle since the cross sections approximately grow as the neutrino energy square, the flux-averaged cross sections can show differences due to the high energy part of the neutrino flux, as discussed in detail for the case of core-collapse supernova neutrino spectra 60 .

Table 2 presents the contribution (in percentage) of the different states to the fluxaveraged cross sections Eq.(1) (15). One can see that in all cases the results for $\gamma=10$ are 

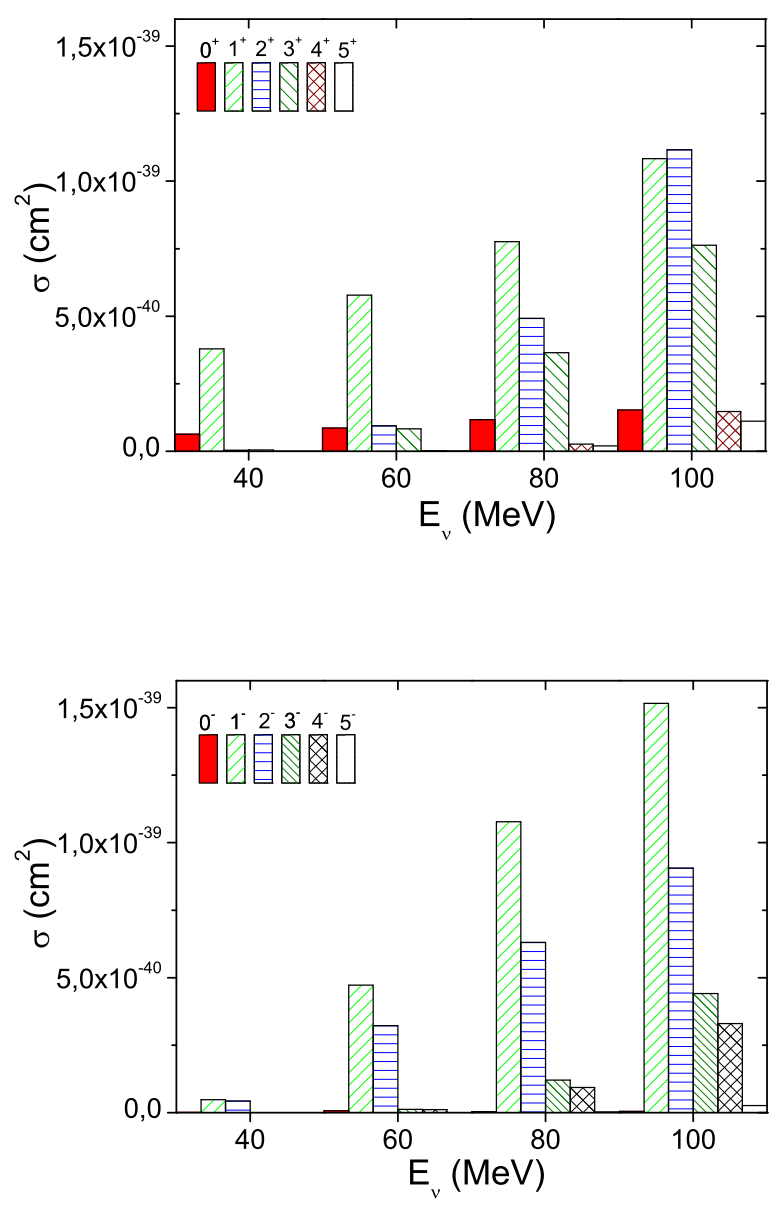

Figure 3. Contribution of the positive (left) as well as negative (right) parity states to the total neutrino-iron cross section, as a function of neutrino energy. 


\begin{tabular}{|lllllll|}
\hline$E_{\nu}(\mathrm{MeV})$ & $\nu^{-208} \mathrm{~Pb}$ & $\bar{\nu}^{2}{ }^{208} \mathrm{~Pb}$ & $\nu_{-}{ }^{100} \mathrm{Mo}$ & $\nu_{-}{ }^{56} \mathrm{Fe}$ & $\bar{\nu}^{-56} \mathrm{Fe}$ & $\nu_{-}{ }^{16} \mathrm{O}$ \\
\hline 7.5 & $2.47(-4)$ & $1.37(-6)$ & $2.30(+0)$ & $8.77(-1)$ & $8.41(-1)$ & - \\
10.0 & $8.49(0)$ & $8.36(-3)$ & $6.84(+0)$ & $3.63(+0)$ & $2.95(+0)$ & - \\
12.5 & $4.68(+1)$ & $7.28(-2)$ & $1.97(+1)$ & $8.94(+0)$ & $6.09(+0)$ & - \\
15.0 & $1.75(+2)$ & $2.44(-1)$ & $4.60(+1)$ & $1.73(+1)$ & $1.03(+1)$ & - \\
20.0 & $8.53(+2)$ & $1.11(0)$ & $2.09(+2)$ & $5.26(+1)$ & $2.17(+1)$ & $7.11(-2)$ \\
25.0 & $2.86(+3)$ & $3.05(0)$ & $5.01(+2)$ & $1.25(+2)$ & $3.74(+1)$ & $5.27(-1)$ \\
30.0 & $4.90(+3)$ & $1.53(0)$ & $9.04(+2)$ & $2.33(+2)$ & $5.74(+1)$ & $2.48(0)$ \\
40.0 & $7.13(+3)$ & $5.65(0)$ & $1.96(+3)$ & $5.44(+2)$ & $1.20(+2)$ & $1.91(+1)$ \\
50.0 & $1.13(+4)$ & $3.48(+1)$ & $3.02(+3)$ & $9.83(+2)$ & $2.09(+2)$ & $7.13(+1)$ \\
60.0 & $1.63(+4)$ & $8.29(+1)$ & $4.67(+3)$ & $1.67(+3)$ & $3.53(+2)$ & $1.85(+2)$ \\
70.0 & $2.20(+4)$ & $1.46(+2)$ & $6.80(+3)$ & $2.59(+3)$ & $5.26(+2)$ & $3.89(+2)$ \\
80.0 & $2.83(+4)$ & $2.16(+2)$ & $9.36(+3)$ & $3.73(+3)$ & $7.27(+2)$ & $6.94(+2)$ \\
90.0 & $3.50(+4)$ & $2.91(+2)$ & $1.23(+4)$ & $5.07(+3)$ & $9.40(+2)$ & $1.10(+3)$ \\
100.0 & $4.16(+4)$ & $3.67(+2)$ & $1.55(+4)$ & $6.60(+3)$ & $1.14(+3)$ & $1.63(+3)$ \\
\hline
\end{tabular}

Table 1

Total cross sections for the indicated neutrino nucleus charged-current reactions as a function of incoming neutrino energy. The cross sections are given in units of $10^{-42} \mathrm{~cm}^{2}$, exponents are given in parentheses.

similar to the DAR case. The neutrino-oxygen cross section is dominated by the $1^{-}, 2^{-}$. Only with increasing $\gamma$, the relative contribution of the $2^{-}$decreases in favor of the positive parity multipoles. This behaviour is due to the oxygen structure: positive parity transitions require at least $2 \hbar \omega$ excitations. The situation is different for the neutrinoiron, neutrino-molybdenum and neutrino-lead cross sections. For these nuclei the $\gamma=6$ case is dominated by the $0^{+}, 1^{+}$transitions, with a relative ratio of about $1 / 3$. When the ion boost increases, their contribution diminishes basically in favor of $1^{-}, 2^{-}$. For $\gamma=14$ the $2^{+}$and $3^{+}$contribution become as important as the Isobaric Analogue state. Table 3 presents the results for the anti-neutrino scattering where the anti-neutrino fluxes are produced by the decay of boosted ${ }^{6} \mathrm{He}$ ions. While the $1^{+}$dominates the flux-averaged cross section at $\gamma=6$ for the iron nucleus, about $50 \%$ of its value is given by the $1^{-}, 2^{-}$ transitions at ion boost 14 . On the contrary, these states dominate at low gamma for the lead nucleus, while almost all multipoles contribute at high gamma. This behaviour can be well understood in terms of the underlying shell structure of the lead nucleus, since the lowest pn-excitations between the filled and unfilled shells are of negative parity.

\section{Conclusions}

We have presented new results on charged-current neutrino-nucleus interactions. The theoretical approach used is the random-phase-approximation among quasi-particles. Four nuclei of particular interest have been chosen as typical examples, namely oxygen, iron, molybdenum, and lead. We have given both the total cross sections as a function of neu- 


\begin{tabular}{|c|c|cccc|cccc|}
\hline${ }^{A} \mathrm{X}$ & & $0^{+}$ & $1^{+}$ & $2^{+}$ & $3^{+}$ & $0^{-}$ & $1^{-}$ & $2^{-}$ & $3^{-}$ \\
\hline${ }^{16} \mathrm{O}$ & $\gamma=6$ & 0.17 & 0.71 & 0.16 & 0.15 & 0.97 & 34.1 & 63.8 & 0.03 \\
& 10 & 0.28 & 1.90 & 1.21 & 1.25 & 0.65 & 43.2 & 51.3 & 0.18 \\
& 14 & 0.43 & 4.04 & 3.36 & 3.33 & 0.34 & 42.4 & 44.8 & 0.75 \\
& $D A R$ & 0.23 & 1.38 & 0.70 & 0.74 & 0.79 & 42.3 & 53.7 & 0.10 \\
${ }^{56} \mathrm{Fe}$ & 6 & 22.6 & 73.1 & 0.16 & 0.19 & 0.03 & 1.6 & 2.32 & 0.0 \\
& 10 & 11.2 & 59.5 & 2.04 & 1.91 & 0.30 & 13.8 & 10.9 & 0.18 \\
& 14 & 6.44 & 39.0 & 6.65 & 5.43 & 0.26 & 23.7 & 15.8 & 1.25 \\
& $D A R$ & 13.0 & 66.7 & 1.09 & 1.12 & 0.24 & 9.45 & 8.27 & 0.06 \\
${ }^{100} \mathrm{Mo}$ & 6 & 22.6 & 69.7 & 0.40 & 0.39 & 0.07 & 2.61 & 4.21 & 0.01 \\
& 10 & 14.6 & 47.3 & 3.24 & 3.16 & 0.28 & 15.6 & 14.5 & 0.66 \\
& 14 & 9.00 & 31.3 & 7.97 & 6.68 & 0.18 & 21.5 & 17.3 & 2.70 \\
& $D A R$ & 16.7 & 53.3 & 2.12 & 2.08 & 0.30 & 12.2 & 12.6 & 0.29 \\
${ }^{208} \mathrm{~Pb}$ & 6 & 21.0 & 62.2 & 1.77 & 1.50 & 0.08 & 2.88 & 10.1 & 0.20 \\
& 10 & 11.8 & 39.3 & 6.63 & 5.91 & 0.24 & 15.1 & 16.8 & 1.82 \\
& 14 & 7.77 & 26.6 & 11.2 & 8.81 & 0.12 & 17.0 & 15.7 & 5.06 \\
& $D A R$ & 12.9 & 43.3 & 4.95 & 4.76 & 0.32 & 14.1 & 17.1 & 1.14 \\
\hline
\end{tabular}

Table 2

Fraction (in \%) of the flux-averaged cross section associated to states of a given multipolarity with respect to the total flux-averaged cross section, i.e. $\langle\sigma\rangle_{J^{\pi}} /\langle\sigma\rangle_{\text {tot }}$. Results are given for all positive and negative states having total angular momentum $J$ between 0 and 5 . The first column gives the considered nucleus, the second tells if the results correspond to low energy beta-beams or to a conventional source $(D A R$ for the decay-at-rest of muons). In the former case the neutrino fluxes are those of ${ }^{18} \mathrm{Ne}$ ions having Lorentz ion boost $\gamma$ between 6 and 14 .

\begin{tabular}{|c|c|cccc|cccc|}
\hline${ }^{A} \mathrm{X}$ & & $0^{+}$ & $1^{+}$ & $2^{+}$ & $3^{+}$ & $0^{-}$ & $1^{-}$ & $2^{-}$ & $3^{-}$ \\
\hline${ }^{56} \mathrm{Fe}$ & 6 & 0.70 & 86.1 & 0.37 & 0.33 & 0.37 & 6.83 & 5.32 & 0.0 \\
& 10 & 0.74 & 51.3 & 3.18 & 2.49 & 0.96 & 26.3 & 14.5 & 0.28 \\
& 14 & 1.01 & 31.0 & 8.95 & 5.61 & 0.68 & 33.9 & 15.7 & 1.61 \\
${ }^{208} \mathrm{~Pb}$ & 6 & 0.57 & 7.04 & 11.8 & 3.82 & 7.21 & 49.9 & 18.7 & 0.60 \\
& 10 & 1.33 & 12.5 & 24.9 & 10.6 & 2.25 & 30.4 & 9.80 & 4.24 \\
& 14 & 1.91 & 13.6 & 25.2 & 9.48 & 0.56 & 18.3 & 9.19 & 10.6 \\
\hline
\end{tabular}

Table 3

Same as Table 2, but for the anti-neutrino nucleus cross sections and the anti-neutrino fluxes produced by boosted ${ }^{6} \mathrm{He}$ ions. 


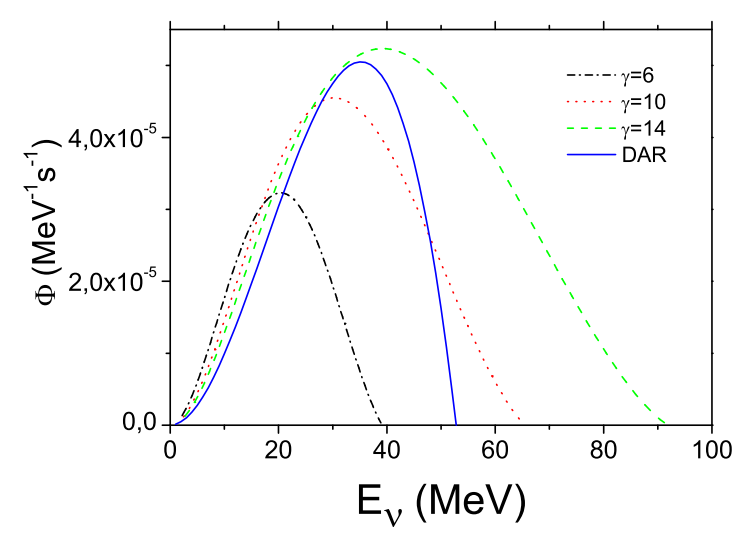

Figure 4. Anti-neutrino fluxes from the decay of ${ }^{6} \mathrm{He}$ ions boosted at $\gamma=6$ (dot-dashed line), $\gamma=10$ (dotted line) and $\gamma=14$ (dashed line). The full line presents the Michel spectrum for neutrinos from muon decay-at-rest.

trino energy and the flux-averaged cross sections associated to possible future facilities producing low energy neutrino beams. These are either based on conventional sources or on low energy beta-beams. A detailed analysis of the states of different multipolarity (allowed and forbidden) contributing to the cross sections has been performed, as a function of the Lorentz ion boosts. The main goal has been to explore if by combining different neutrino ion accelerations, information on the various multipoles can be extracted. We conclude that by varying the $\gamma$ factor, the role of the spin-dipole states becomes as important as the Isobaric Analogue and Gamow-Teller states allowing nuclear structure studies of the forbidden states.

We acknowledge the financial support of the EC under the FP6 "Research Infrastructure Action - Structuring the European Research Area' EURISOL DS Project, Contract Number 515768 RIDS. 


\section{REFERENCES}

1. K. Muto, E. Bender and H.V. Klapdor, Z. Phys. A 334, 177 (1989); M. Aunola, J. Suhonen, Nucl. Phys. A 602, 133 (1996).

2. M. Kortelainen and J. Suhonen, Europhys. Lett. 58, 666 (2002) arXiv:nucl-th/0201007.

3. M. Kortelainen and J. Suhonen, J. Phys. G 30, 2003 (2004).

4. V. A. Rodin, A. Faessler, F. Simkovic and P. Vogel, Phys. Rev. C 68, 044302 (2003) arXiv:nucl-th/0305005.

5. H. Akimune et al., Phys. Lett. B 394, 23 (1997); J. Bernabeu et al., IPNO/TH-88-58, FTUV-88/20.

6. S. Rakers et al., Phys. Rev. C 70, 054302 (2004).

7. S. Rakers et al., Phys. Rev. C 71, 054313 (2005).

8. C. Volpe, J. Phys. G 31, 903 (2005) arXiv:hep-ph/0501233.

9. J. Engel, M. Bender, J. Dobaczewski, W. Nazarewicz and R. Surman, Phys. Rev. C 60, 014302 (1999) arXiv:nucl-th/9902059.

10. I. N. Borzov and S. Goriely, Phys. Rev. C 62, 035501 (2000).

11. A. Heger, E. Kolbe, W. C. Haxton, K. Langanke, G. Martinez-Pinedo and S. E. Woosley, Phys. Lett. B 606, 258 (2005) arXiv:astro-ph/0307546].

12. B. S. Meyer, G. C. McLaughlin and G. M. Fuller, Phys. Rev. C 58, 3696 (1998) arXiv:astro-ph/9809242].

13. R. Surman and J. Engel, Phys. Rev. C 58, 2526 (1998) arXiv:nucl-th/9805007].

14. K. Langanke and G. Martinez-Pinedo, Rev. Mod. Phys. 75, 819 (2003) arXiv:nucl-th/0203071.

15. A. B. Balantekin and G. M. Fuller, J. Phys. G 29, 2513 (2003) [arXiv:astro-ph/0309519].

16. M. Bender, J. Dobaczewski, J. Engel and W. Nazarewicz, Phys. Rev. C 65, 054322 (2002) arXiv:nucl-th/0112056] and references therein.

17. F. Osterfeld, Rev. Mod. Phys. 64, 491 (1992)and references therein.

18. E. Caurier, G. Martinez-Pinedo, F. Nowacki, A. Poves and A. P. Zuker, Rev. Mod. Phys. 77, 427 (2005) arXiv:nucl-th/0402046 and references therein.

19. A. Bobyk, W.A. Kaminski, F. Simkovic, Phys. Rev. C 63, 051301 (2001); J. Suhonen, J. Phys. G 19, 139 (1993); K. Muto, E. Bender and H.V. Klapdor-Kleingrothaus, Z. Phys. A 339, 435 (1991); K. Muto, E. Bender and H.V. Klapdor-Kleingrothaus, Z. Phys. A 334, 187 (1989); T. Tomoda and A. Faessler, Phys. Lett. B 199, 475 (1987).

20. O. Civitarese and J. Suhonen, Phys. Lett. B 626, 80 (2005).

21. K. Yako, H. Sagawa and H. Sakai, Phys. Rev. C $\mathbf{7 4}$ (2006) 051303 arXiv:nucl-ex/0609046].

22. N. T. Zinner, K. Langanke and P. Vogel, Phys. Rev. C 74, 024326 (2006) arXiv:nucl-th/0606002 and references therein.

23. Krasznahorkay et al., Phys. Rev. Lett. 82 (1999) 3216.

24. S. E. Willis et al., Phys. Rev. Lett. 44, 522 (1980) [Erratum-ibid. 44, 903 (1980 ERRAT, 45,1370.1980)].

25. B. Zeitnitz et al., Prog. Part. Nucl. Phys. 40, 169 (1998).

26. C. Athanassopoulos and the LSND collaboration, Phys. Rev. C 56, 2806 (1997) 2806; 
M. Albert et al, Phys. Rev. C 51, R1065 (1995); C. Athanassopoulos and the LSND collaboration, Phys. Rev. C 55, 2078 (1997) ; D.A. Krauker et al, Phys. Rev. C 45, 2450 (1992) ; R.C. Allen et al, Phys. Rev. Lett. 641871 (1990) ; B.E. Bodmann and the KARMEN collaboration, Phys. Lett. B 332, 251 (1994) ; J. Kleinfeller in Neutrino 96, eds. K.Enquist, H.Huitu and J.Maalampi (World Scientific Singapore, 1997).

27. E. Kolbe et al, Phys. Rev. C 52, 3437 (1995); N. Auerbach, N. Van Giai and O.K. Vorov, Phys. Rev. C 56, R2368 (1997); S.K. Singh, N.C. Mukhopadyhay and E. Oset, Phys. Rev. C 57, 2687 (1998); S.L. Mintz and M. Pourkaviani, Nucl. Phys. A 594, 346 (1995); E. Kolbe, K. Langanke and P. Vogel, Nucl. Phys. A 613, 382 (1997); A.C. Hayes and I.S. Towner, Phys. Rev. C 61, 044603 (2000); C. Volpe et al, Phys. Rev. C 62, 015501 (2000) ; N. Auerbach and B.A. Brown, Phys. Rev. C 65, 024322 (2002).

28. N. Jachowicz, K. Heyde, J. Ryckebusch and S. Rombouts, Phys. Rev. C 65, 025501 (2002).

29. E. Kolbe, K. Langanke and P. Vogel, Phys. Rev. D 66, 013007 (2002).

30. G. M. Fuller, W. C. Haxton and G. C. McLaughlin, Phys. Rev. D 59, 085005 (1999) arXiv:astro-ph/9809164.

31. E. Kolbe and K. Langanke, Phys. Rev. C 63, 025802 (2001) arXiv:nucl-th/0003060].

32. C. Volpe, N. Auerbach, G. Colo and N. Van Giai, Phys. Rev. C 65, 044603 (2002) arXiv:nucl-th/0103039.

33. M. Sajjad Athar, S. Ahmad and S. K. Singh, Nucl. Phys. A 764, 551 (2006) arXiv:nucl-th/0506046.

34. F. T. Avignone and Y. V. Efremenko, J. Phys. G 29, 2615 (2003).

35. P. Zucchelli, Phys. Lett. B 532,166 (2002).

36. C. Volpe, J. Phys. G 30, L1 (2004) arXiv:hep-ph/0303222.

37. J. Serreau and C. Volpe, Phys. Rev. C 70, 055502 (2004) [arXiv:hep-ph/0403293].

38. G. C. McLaughlin, Phys. Rev. C 70, 045804 (2004) |arXiv:nucl-th/0404002|.

39. N. Jachowicz and G. C. McLaughlin, Phys. Rev. Lett. 96, 172301 (2006) arXiv:nucl-th/0604046.

40. G. C. McLaughlin and C. Volpe, Phys. Lett. B $591 \quad$ (2004) 229 arXiv:hep-ph/0312156.

41. A. B. Balantekin, J. H. de Jesus and C. Volpe, Phys. Lett. B 634, 180 (2006) arXiv:hep-ph/0512310.

42. A. B. Balantekin, J. H. de Jesus, R. Lazauskas and C. Volpe, Phys. Rev. D 73, 073011 (2006) arXiv:hep-ph/0603078.

43. A. Bueno, M. C. Carmona, J. Lozano and S. Navas, Phys. Rev. D 74, 033010 (2006).

44. J. Barranco, O. G. Miranda and T. I. Rashba, arXiv:hep-ph/0702175.

45. C. Volpe, J. Phys. G 34, R1 (2007) arXiv:hep-ph/0605033|.

46. P. S. Amanik and G. C. McLaughlin, arXiv:hep-ph/0702207.

47. R. Lazauskas, A. B. Balantekin, J. H. De Jesus and C. Volpe, arXiv:hep-ph/0703063.

48. C.K. Jung, Proceedings of the Next generation Nucleon Decay and Neutrino Detector (NNN99) Workshop, September 23-25, 1999, Stony Brook, New York hep-ex/0005046.

49. A. de Bellefon et al., arXiv:hep-ex/0607026.

50. Itow et al., hep-ex/0106019.

51. H. Ejiri, J. Engel, R. Hazama, P. Krastev, N. Kudomi and R. G. H. Robertson, Phys. 
Rev. Lett. 85, 2917 (2000) arXiv:nucl-ex/9911008.

52. S.R. Elliott, Phys. Rev. C 62, 055501 (2000); P.F. Smith, Astropart. Phys. 8, 27 (1997); C.K. Hargrove et al, Astropart. Phys. 5, 183 (1996); D.B. Cline et al, Phys. Rev. D. 50, 720 (1994).

53. N. Jachowicz, K. Heyde and J. Ryckebusch, Phys. Rev. C 66, 055501 (2002).

54. J.D. Walecka, Muon Physics, ed. V.M. Hughes and C.S Wu (Academis, New York, 1975).

55. J. Engel, Phys. Rev. C 57, 2004 (1998) arXiv:nucl-th/9711045.

56. A. Chancé and J. Payet, Private communication.

57. E. Kolbe, K. Langanke and G. Martinez-Pinedo, Phys. Rev. C 60, 052801 (1999) arXiv:nucl-th/9905001.

58. S. L. Mintz, J. Phys. G 28, 451 (2002).

59. T. Suzuki and H. Sagawa, Nucl. Phys. A 718 (2003) 446c.

60. N. Jachowicz and K. Heyde, Phys. Rev. C 68, 055502 (2003). 